\title{
Urethral duplication in male children's: report of four cases
}

\author{
Abstract \\ Objective: To evaluate the medium-term outcomes of the management of children \\ with urethral duplication.
}

Materials and Methods: We report a retrospective study of four children carrying urethral duplication followed in our department over a period of 5 years from 2010 to 2015; Preoperative exploration was based on ultrasound, urethrocystography retrograde and the fistulography.

Results: In our study, three patients had rare form of epispadias urethral duplication (complete type II of Effmann and Leibowitz) and one, "Y" duplication (type II A2). We proceeded in all cases, to the resection of a super urethra excision. The follow up was from 6 months to 2 years for our patients. There was no infectious complication. For one patient with Y urethral duplication, a stenosis of the path of the dorsal urethra was preserved within complete urination.

Conclusion: Urethral duplication on a boy is a rare anomaly of the urinary tract. The diagnosis and the determination of its type are based on the retrograde cryptography. Its varied clinical expression depends on its anatomical type. If the diagnosis is established in a symptomatic context, the surgical indication is posed with good performances in most cases.
Volume I Issue 4 - 2017

\author{
Achraf El Bakkaly, Toualouth Lafia, Jaouad \\ Bouljrouf, Mohammed Rami, Rachid \\ Belkacem, Mohammed Amine Bouhafs \\ Department of Pediatric Urology, University Hospital of lbn \\ Sina, Morocco
}

\begin{abstract}
Correspondence: Achraf El Bakkaly, Resident doctor in Department of Pediatric Urology "C", Children's Hospital, University Hospital of Ibn Sina, Faculty of Medicine Mohammed V, BP 6527, Street Lamfadel Cherkaoui Rabat Institute, Rabat Morocco, Email achraf.elbakkalyl@gmail.com
\end{abstract}

Received: August 26, 2017| Published: December 12, 2017

Keywords: accessory urethra, duplication, malformation

\section{Introduction}

The urethral duplication or supernumerary urethra or called accessory urethra is an exceptional malformative uropathy of interest mainly to the boy. ${ }^{1}$ It is defined by the juxtaposition of 2 or more channels with smooth muscular structure with a mucosal coating of excreto-urinary type. ${ }^{2}$ It can be complete or incomplete. ${ }^{3}$ It occurs preferentially in the sagittal plane but also in the frontal plane. ${ }^{2}$ Numerous anatomical varieties have been described with a second urethra opening in normal position or not. The therapeutic attitude can be complex, depending on the anatomical type. ${ }^{1}$ Radiological investigations are of great interest in all cases. ${ }^{4}$ We report four cases of urethral duplication in the boy. Our objective through this work is an assessment of the medium-term outcomes of the management of children with urethral duplication.

\section{Materials and methods}

We retrospectively reviewed the records of four male patients treated for urethral duplication during five years, from January 2010 to December 2015. The age at diagnosis varied from 2 years to 11 years. For all our patients, the paraclinic evaluation included ultrasound, retrograde urethrocystography and urinary endoscopy.

\section{Observation $n^{\circ} \mathbf{I}$}

A male infant, 2 years, was admitted for perineal fistula with an apical meatus in normal place (Figure 1). The clinical examination under general anesthesia found a small fistula of 1 to $2 \mathrm{~mm}$ in diameter, at the perineo scrotal junction, at 12 o'clock from the anal orifice, letting a few drops of urine during urination. A good apical urinary stream was observed. Retrograde urethrocystography supplemented by endoscopy showed a fistulous pathway from the prostatic urethra to the scrotum. This pathway corresponded to a supernumerary "Y" urethra coming from posterior urethra.

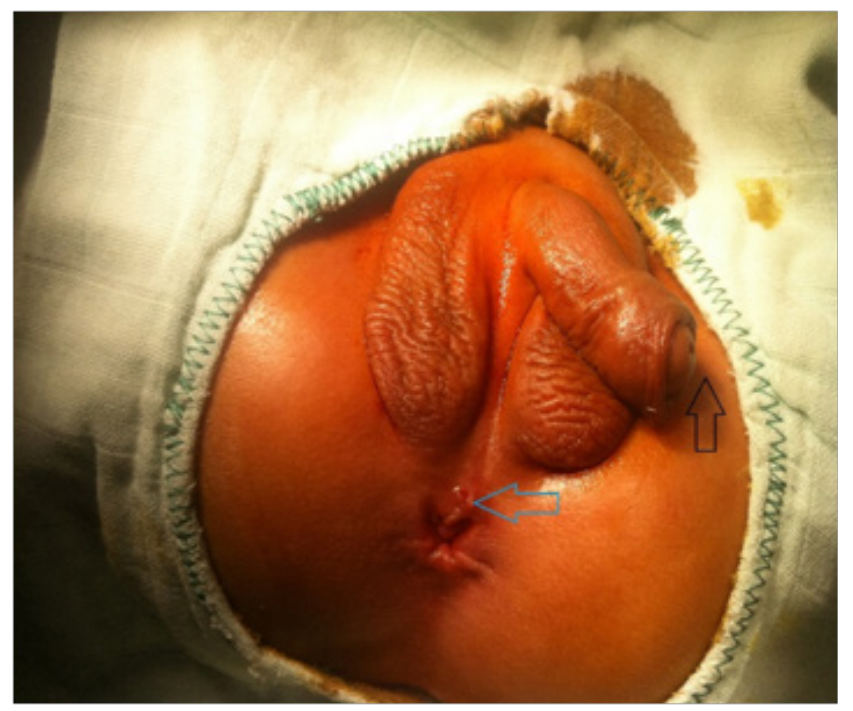

Figure I Perineal urethral fistula with peeling of urine drops (blue arrow); The penis with a single cavernous body and a light meatus filiform epispadic impossible to catheterize (black arrow).

The patient has been operated at two and a half years. The exploration proved that it was rather a urethral fistula and not a real urethra because the path was very fine. A complete resection of this urethral fistula was made after failed attempts to dissect the path and bring it into distal. 


\section{Observation $n^{\circ} 2$}

Boy of 4 years old, admitted for a dual urinary stream through two separate orifices. The clinical examination found a child circumcised with a genital malformation, supernumerary urethra epispadic that was entered on the dorsal aspect of the penis with a bend of the penis. Plain $x$-ray of the abdomen showed a disjunction of the pubic symphysis. Ultrasound was unusual. Retrograde urethrocystography showed a good-capacity bladder with a lateral ureterocele, the urethra seems to have normal morphology. The patient was operated, a catheterization under general anesthesia made it possible to follow the two orifices: the main and ventral meatus, letting flow urine (Figure 2). A meticulous dissection of the epispadic meatus was done to its origin in the bladder, revealing two totally independent urethras. The difficulty of the dissection of the second urethra until its confusion with the bladder led us to realize a bladder cut to check the integrity of the excision. The supernumerary urethra was excised with partial derotation of the penis. Finally, a dorsal plasty of the penis has been done either a correction of the curvature of the penis (Figure 3 ).

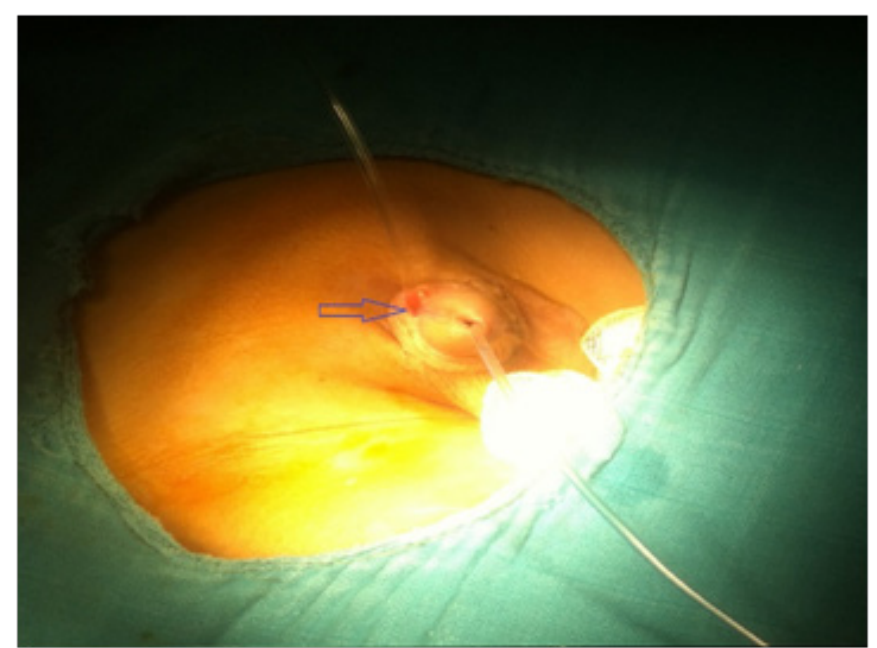

Figure 2 Survey of the 2 urethral meats in our patient (Blue arrow: epispadic dorsal meatus).

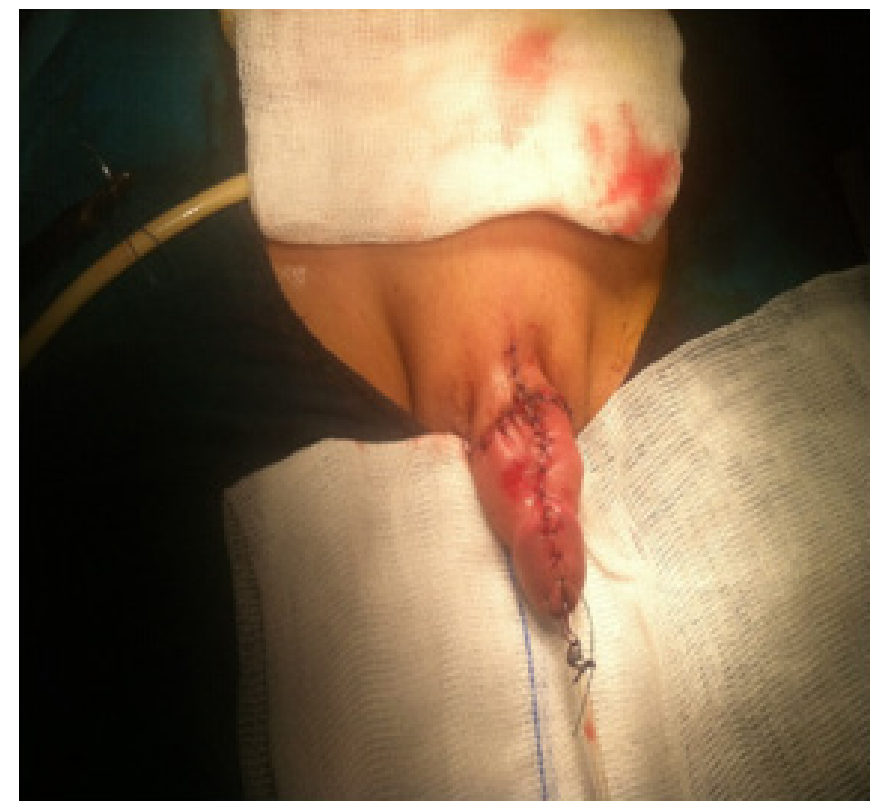

Figure 3 Final appearance of the penis.

\section{Observation $\mathrm{n}^{\circ} 3$}

Boy of 7 years consults for emission of urine by 2 urethral meats. Clinical examination found an accessory epispadic urethra. Ultrasound of the urinary tract and retrograde urethrocystography were normal. Fistulography has objectified a complete accessory urethra communicating with the bladder and opening up over the bladder neck. The patient was operated on and the fistula was excised with the rectification of the penis.

\section{Observation $n^{\circ} 4$}

Boy of 11 years was admitted for emission of the urines by 2 orifices. The clinical examination found an epispadic supernumerary urethra which was inserted on the dorsal surface of the penis. Ultrasound of the urinary tract and retrograde urethrocystography were normal. Fistulography has objectified a complete accessory urethra entering the bladder. An excision of the supernumerary urethra was performed with plasty of the penis

\section{Results}

According to the classification of Effmann \& Leibowitz ${ }^{5}$ (Figure 4), the group of our patients is group II which integrates complete or incomplete urethral duplications which are subdivided into type IIA in which there are two meats with subtype IIA1 (three of Our patients): Two independent ureters originating from the bladder and for subtype IIA2 (one patient): two ureters joined together before bladder penetration or Y duplication (Table 1).

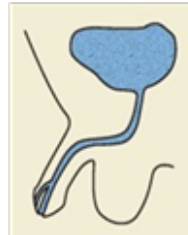

IA

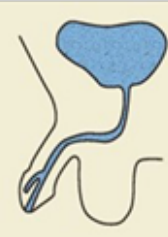

IB

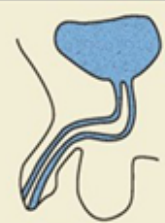

IIA 1

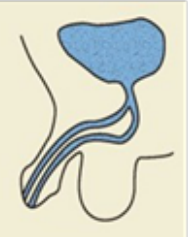

IIA 2

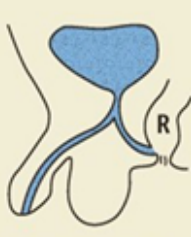

IIA 2 "Y type"

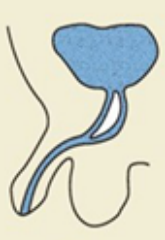

IIB

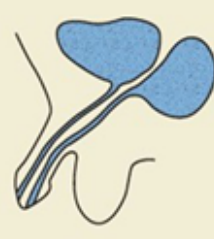

III
Figure 4 Classification of Effmann and Leibowitz of urethral duplication in child.5

For the first case, the evolution was marked by a stenosis of the path. The child was re-admitted to the operating room for exploration and attempted re-sealing of the fistula. The conserved dorsal urethra allowed incomplete urination with a very fine urinary stream. The penis had a single corpus cavernosum, flaccid to the test of simulation of erection. The child currently urinates by the penis and has been vesicostomized at the age of three years. He is expected to have a continent urinary derivation type Mitrofanoff. For the other three cases, the evolution after 2 years of hindsight is good. Children were continents even to the stress with a good urine stream. The aesthetic appearance of the penis was acceptable. To evaluate the results of these 3 patients, we adopted the HOPE score which evaluates the position of the meatus, its shape, the shape of the glans, the penile skin as well as the existence of a curvature of the penis during erection. ${ }^{6}$ We obtained in all cases a good result between 40 and 50 points. 
Table I Description of our cases of duplication of the urethra: age at diagnosis/ age at intervention/ operating technique/ followed (month)/ evolutioncomplications

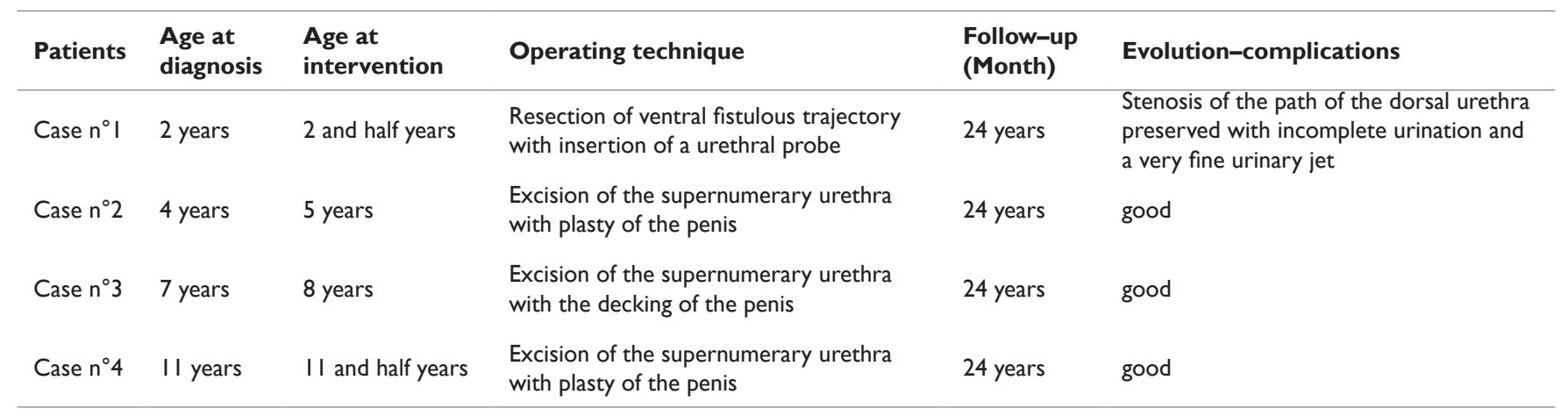

\section{Discussion}

Duplication of the urethra is a rare malformation. ${ }^{7}$ It is seen mostly in the boy and takes several anatomical forms: hypospadias (the two urethra are situated beneath the cavernous bodies), epispadias (the supernumerary urethra is situated above the cavernous bodies) and posterior $\mathrm{Y}$-forms with Perineal or anal meatus. ${ }^{3,5,8}$

Three of our cases are considered to be Type II AI of the Effmann and Leibowitz classification: this is a complete duplication. This is the group most frequently encountered in the majority of the literature series. ${ }^{1,9,10}$ The ventral urethra emerging from the bladder opens from the region of the glans to the penoscrotal region. The ventral urethra is always the most functional of the two, although the dorsal urethra is the only one in the normal position. ${ }^{1,2}$ The age of discovery is precocious, usually before the age of one $y e a r,{ }^{2}$ a case of our patients was diagnosed at 24 months.

Urethral duplication is often of fortuitous discovery on examination of the external genitalia of the newborn. It is essentially asymptomatic, ${ }^{9}$ but if it is symptomatic the signs commonly encountered are the existence of a double urinary jet, repeated urinary infections, dysuria and urinary incontinence. ${ }^{7}$ The association with other anomalies of the upper urinary tract is rare. Genito-urinary malformations (renal dysplasia, vesicoureteral reflux, bladder exfoliation, cryptorchidia), digestive and musculoskeletal disorders have been described., ${ }^{2,7}$ This is the case of our observation No. 1 which had a single corpus cavernosum. Cystography studies the anomaly. Coupled with endoscopy, cystography makes it possible to better appreciate the anatomical aspect and especially the functional urethra ${ }^{11}$ and it evaluates its continence. The management of urethral duplication involves several techniques, ranging from sclerosing injections of the supernumerary canal to surgical excision of the accessory urethra or genito-urinary reconstruction surgery of the hypospadias. ${ }^{8,12-14}$

In types IIA1 (the case of three of our patients), the treatment of the supernumerary urethra is not yet well codified and the therapeutic attitude remains variable from one author to another. Only symptomatic forms are operated. ${ }^{15}$ Before any surgical procedure, it is imperative to specify the anatomy and the path of the 2 ureters. The excision of the dorsal urethra can be successfully performed by a double perineal and pre-pubic approach. ${ }^{16}$ The total excision of the accessory urethra is ideal ${ }^{8,9}$ with liberation of the corpus cavernosum when there is a dorsal curvature at the erection; It is delicate because it must take account of the presence of the erector nerves and the external sphincter. Our results perfectly corroborate those encountered by several authors of the literature using the same surgical procedure. Merrot et al. ${ }^{1}$ in his heterogeneous series of urethral sagittal duplications had two cases of epispade duplications with complete resection of the accessory meatus. Jouini et al. ${ }^{17}$ performed a complete removal of the supernumerary urethra with putting upright the penis to treat the only case of urethral duplication epispade of its series.

The management of urethral duplication in $\mathrm{Y}$ is very controversial. Indeed, if the ventral urethra is functional, which has not been our case, excision of the supernumerary urethra can be successfully considered. ${ }^{18,19}$ This is the case of Y-duplication in a 14-year-old boy successfully treated by Sanchez, ${ }^{4}$ with complete dissection and ligation of the supernumerary urethra through a perineal approach. According to the author, duplication in $\mathrm{Y}$ is a benign pathology and therefore during surgery it is important to recognize and preserve the functional urethra in order to avoid potentially serious sphincter complications. However, if the ventral urethra is hypoplastic as in our observation $\mathrm{n}^{\circ} 1$, after excision, urethroplasty by a vascularized tubular flap is proposed by some authors. ${ }^{20}$ Podesa \& Ortolano ${ }^{13,20}$ proposed the use of free grafts of oral mucosa. In our case, it is practically impossible to perform these urethroplasty procedures because dissection should begin at the prostatic urethra. Another therapeutic alternative for the management of urethral duplication in $\mathrm{Y}$ is proposed by Lima and colleagues in his recent study based on the evaluation of 40 years of management of eight patients with urethral duplication in $\mathrm{Y}^{21}$ The authors conclude that it would be safer and more efficient to treat this type of duplication by trans-rectal sagittal approach (ASTRA), thus allowing the excision of the ectopic urethra and for urethroplasty they propose the use of a flap Free of bladder mucosa with colostomy preventing infectious complications. ${ }^{22}$

\section{Conclusion}

Urethral duplication in the boy is a rare anomaly. Its clinical expression depends on its anatomical type. In patients with a dual urinary stream, the evaluation will be complete only if both ureters are identified in their entirety. ${ }^{9}$ If the diagnosis is made in a symptomatic context, the surgical indication is made with good results in most cases. Our work illustrates the severity of some cases of Y duplication especially if associated with other malformations of the penis (a single corpus cavernosum) and the difficulty of management requiring several surgical procedures with an average cosmetic result.

\section{Acknowledgements}

None.

\section{Conflict of interest}

The author declares no conflict of interest. 


\section{References}

1. Merrot T, Weber D, Steyeart H, et al. Duplications sagittales de l'urètre chez l'enfant. A propos de 7 observations. Progrès en Urologie. 2002;12(1):77-83.

2. Bouhafs A, Abarchi H, Belkacem R, et al. Urethral anterior duplication in male. A case report. Annales d'Urologie. 2002;36(2):146-149.

3. Williams DI, Kenawi MM. Urethral duplication in the male. Eur Urol. 1985;1(5):209-215.

4. Sánchez MM, Vellibre RM, Castelo JL, et al. A new case of male Ytype urethral duplication and review of literature. J Pediatr Surg. 2006;41(1):e69-e71

5. Effmann EL, Lebowitz RL, Colodny AH. Duplication of the urethra Radiology. 1976;119(1):179-185.

6. Van der Toorn F, De Jong TP, De Gier RP, et al. Introducing the HOPE (Hypospadias Objective Penile Evaluation)-score: A validation study of an objective scoring system for evaluating cosmetic appearance in hypospadias patients. J Pediatr Urol. 2013;9(6):1006-1017.

7. Tillig B, Möritz RP, Müller W, et al. Duplication of the male urethra: Two case reports. Eur J Pediatr Surg. 1999;9(3):189-192.

8. Lima M, Destro F, Maffi M, et al. Practical and functional classification of the double urethra: A variable, complex and fascinating malformation observed in 20 patients. J Pediatr Urol. 2017;13(1):42.e1-42.e7.

9. Salle JL, Sibai H, Rosenstein D, et al. Urethral duplication in the male: review of 16 cases. J Urol. 2000;163(6):1936-1940.

10. Abou Zeid AA, Mohammad SA, Radwan NA, et al. Y-type urethral duplication: a true variant of the anomaly or a misnomer? Eur J Pediatr Surg. 2015;26(3):245-251
11. Moulsdale JE, Marshall FF. Partial duplication of the male urethra. $J$ Urol. $1977 ; 118(2): 336-338$

12. Belman $\mathrm{AB}$. The repair of a congenital $\mathrm{H}$-Type urethrorectal fistula using a scrotal flap urethroplasty. J Urol. 1977;118(4):659-661.

13. Ortolano V, Nasrallah PF. Urethral duplication. J Urol. 1986;136(4):909_ 912 .

14. Rodríguez Tolrá J, Cuadrado Campaña JM, Buisan O, et al. Duplicidad uretral incompleta. Actas Urol Esp. 2005;29(7):697-699.

15. Stephens FD, Donnellan WL. "H-type" urethroanal fistula. J Pediatr Surg. 1977;12(1):95-102.

16. Levin TL, Han B, Little BP. Congenital anomalies of the male urethra. Pediatr Radiol. 2007;37(9):851-862.

17. Jouini R, Krichéne I, Lefi M, et al. Urethral duplication in male childrens: report of 4 cases. Annales d'urologie. 2003;37(5):288-292.

18. Holst S, Peterson NE. Fulguration-ablation of atypical accessory urethra. J Urol. 1988;140(2):347-348.

19. Wagner JR, Carr MC, Bauer SB, et al. Congenital posterior urethral perineal fistulae: a unique form of urethral duplication. Urology. 1996;48(2):277-280.

20. Davidson JR, Wright NJ, Garriboli M. Urethral Duplication with Two Hypospadic Meati-An Unusual Variant. European J Pediatr Surg Rep. 2016;4(1):37-40

21. Podesta ML, Medel R, Castera R, et al. Urethral duplication in children: surgical treatment and results. J Urol. 1998;160(5):1830-1833.

22. Lima M, Destro F, Di Salvo N, et al. Fate of males with urethral "Yduplication":40-year long follow-up in 8 patients. J Pediatr Surg. 2016;52(8):1335-1339. 